\title{
On Cell-Matrix Interactions in Mammary Gland Development and Breast Cancer
}

\author{
Rabih Talhouk \\ Department of Biology, American University of Beirut, Beirut, Lebanon \\ Correspondence: rtalhouk@aub.edu.lb
}

The he review by Muschler and Streuli (2011) elegantly highlights the remarkable strides made in understanding the role of extracellular matrix (ECM) in regulating the different stages of mammary development and breast cancer. Since the early 1970s the dogma that ECM regulates tissue function as well as the conceptual notion that the cell and its microenvironment, including cell-ECM interaction, cell-cell interaction, and soluble signaling, constitute the smallest unit of function persisted. However, the majority of studies have focused on how ECM and soluble mediators, together or separately, modulate cell-specific function, whereas relatively less attention was given to the role of cell-cell interaction. More so, compound modes of signaling added to the intricacy of cell-ECM and soluble signaling cascades. Examples of such signaling modes include receptor transactivation, whereby integrin-dependent adhesion can transactivate, via ligand-independent mechanism, neighboring growth factor receptors. Another is the "sink effect" of ECM in which soluble mediators, such as growth factors, bind to the ECM, which then, in reciprocity with the cell, modulate how they are presented to the cell and when (Patel et al. 2007; Streuli and Akhtar 2009). Such compound modes of signaling and cooperation between integrins and other receptor systems, and recent literature on cellcell interaction (Dbouk et al. 2009; Lelievre 2010) all point to the fact that signaling needs to be studied within complex microenvironments and in proper tissue context.

Intercellular junctions are no longer perceived as simple physical barriers, structural scaffolds, or channels that contribute to proper tissue architecture and homeostasis, but rather as active contributors to signal transduction emanating from the complex of proteins assembling at the junctional hub. We have shown that mammary epithelial cell differentiation is dependent on the assembly of the gap junction protein complex at the membranes of apposing cells. This complex, which is modulated by the type of ECM and coculture conditions includes, under optimal differentiation-permissive conditions, catenin and zonula adherens proteins in association with the full complement of mammary connexins. The assembly of the complex was concomitant with the recruitment of $\beta$-catenin from the nucleus to the gap junction complex. Disruption of the gap junction complex leads to a loss of differentiated phenotype (Talhouk et al. 2008). Others, and we, are engaged in deciphering the role of the junction reassembly in MDA-MB231 breast cancer cell lines that typically lack membrane connexin and junctional assemblies. By reintroducing and overexpressing connexin- 43 , the cells reverted, in a context-dependent manner, toward a lesser tumorigenic phenotype. In addition, Laird's group has shown a connexin-43 tumor-suppressive effect in keratinocytes that may be linked to its interaction

Editors: Mina J. Bissell, Kornelia Polyak, and Jeffrey M. Rosen

Additional Perspectives on The Mammary Gland as an Experimental Model available at www.cshperspectives.org

Copyright (C) 2012 Cold Spring Harbor Laboratory Press; all rights reserved; doi: 10.1101/cshperspect.a013540

Cite this article as Cold Spring Harb Perspect Biol 2012;4:a013540 
R. Talhouk

with a tumor-suppressing protein caveolin-1 (Naus and Laird 2010).

Similarly, apical polarity regulators (Crb complex, Par complex, and Scrib, Dlg, and Lgl proteins) and tight junction proteins, claudin and occludin, and their associated proteins on one hand modulate signal transduction and distribution of transcription regulators between nuclear compartment and tight junction complexes and, on the other, participate in modulating chromatin-associated complexes. This relationship between tight junction assembly, apical polarity, and chromatin architecture might explain how cells become cancerous, following alterations or loss in apical polarity (Lelievre 2010).

Despite all of these advances, much of our knowledge today still depends on the use of culture-based systems that lack many of the components needed for cells to assemble into morphologies that resemble their in vivo counterparts. More so, many culture models use systems initially deficient in expression of the protein of interest, and as such the functions of several proteins are studied out of their optimal tissue context. Thus, there is a need for more physiologically relevant model systems that can faithfully recapitulate the tissue context in a $3 \mathrm{D}$ setting and beyond, recognize the importance of self-assembly and self-organizing principles of cells, and allow for timely delivery of the component parts (cells, soluble mediators, and ECM), so that tissues and organs are reconstructed de novo. Future efforts should aim to construct culture models that culminate, beyond the sum of its individual parts, in reproducing organ function. In this respect, microfluidic devices and bioprinting have the potential to reconstitute the interactions in vitro, in physiologically relevant milieu, to address cell-ECM interactions in the proper context of cell-cell microenvironmental cues and vice versa.

Our understanding of signal transduction whether via ECM, neighboring cells, and/or soluble mediators will definitely evolve as we continue to integrate the different aspects that make up the tissue context, and as we increase our knowledge of the organizing principles governing cell, tissue, organ, and ultimately organism function. Despite all of the progress witnessed so far, many challenges as well as exciting discoveries lay ahead.

\section{REFERENCES}

${ }^{*}$ Reference is also in this collection.

Dbouk HA, Mroue M, El-Sabban ME, Talhouk RS. 2009. Connexins: A myriad of functions extending beyond assembly of gap junction channels. Cell Commun Signal 7: 4 .

Lelièvre SA. 2010. Tissue polarity-dependent control of mammary epithelial homeostasis and cancer development: An epigenetic perspective. J Mammary Gland Biol Neoplasia 15: 49-63.

${ }^{*}$ Muschler J, Streuli CH. 2011. Cell-matrix interactions in mammary gland development and breast cancer. Cold Spring Harb Perspect Biol doi: 10.1101/cshperspect. a003202.

Naus CC, Laird DW. 2010. Implications and challenges of connexin connections to cancer. Nat Rev Cancer 10: 435-441.

Patel VN, Knox SM, Likar KM, Lathrop CA, Hossain R, Eftekhari S, Whitelock JM, Elkin M, Vlodavsky I, Hoffman MP. 2007. Heparanase cleavage of perlecan heparan sulfate modulates FGF10 activity during ex vivo submandibular gland branching morphogenesis. Development 134: $4177-4186$

Streuli CH, Akhtar N. 2009. Signal co-operation between integrins and other receptor systems. Biochem J 418: 491-506.

Talhouk RS, Mroue RM, Mokalled M, Abi-Mosleh L, Nehme R, Ismail A, Zaatari M, Khalil A, El-Sabban ME. 2008. Heterocellular interaction enhances recruitment of $\alpha$ and $\beta$-catenins and ZO-2 into functional gap junction complexes and gap junction-dependent differentiation of mammary epithelial cells. Exp Cell Res 314: $3275-3291$ 


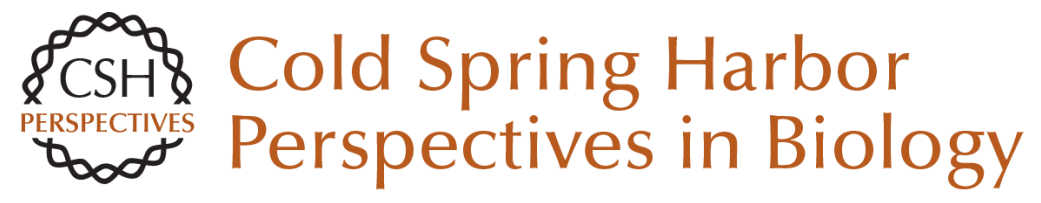

\section{On Cell-Matrix Interactions in Mammary Gland Development and Breast Cancer}

Rabih Talhouk

Cold Spring Harb Perspect Biol 2012; doi: 10.1101/cshperspect.a013540

Subject Collection

For additional articles in this collection, see http://cshperspectives.cshlp.org/cgi/collection/

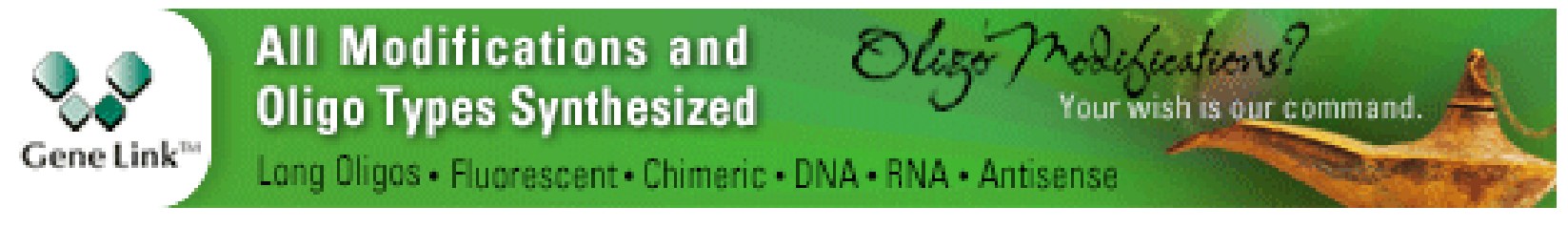

Copyright @ 2012 Cold Spring Harbor Laboratory Press; all rights reserved 